\title{
Somatostatinoma de duodeno: relato de caso e revisão da literatura
}

Duodenal somatostatinoma: case report and literature review

Alexandre Cruz Henriques', Ana Maria Antonio Mader², Giselle Munhoz Ramos'1, Rafaela Rosalba de Mendonça', Sergio Renato Pais Costa', Leandro Luongo de Matos', Manlio Basílio Sperazini'

\begin{abstract}
Resumo
Introdução: Somatostatinomas são tumores carcinóides produtores de somatostatina, localizados principalmente na cabeça do pâncreas e duodeno. A importância desse relato deve-se à raridade deste subtipo histológico. Relato de Caso: Mulher, 68 anos, portadora de diabetes mellitus há dois anos, com queixa de dor abdominal há cinco meses. Ao exame físico notava-se massa em epigástrio e hipocôndrio direito, fixa e dolorosa à palpação. A ultra-sonografia e a tomografia computadorizada de abdome evidenciaram, respectivamente, colelitíase e tumor sólido em topografia de cabeça de pâncreas, medindo cerca de $12 \times 8 \mathrm{~cm}$. A paciente foi submetida a procedimento cirúrgico, no qual foi encontrado um grande tumor envolvendo o duodeno e o pâncreas, optando-se pela gastroduodenopancreatectomia cefálica e colecistectomia. Os exames anátomo-patológico e imuno-histoquímico da peça operatória revelararam tratar-se de um somatostatinoma de duodeno (estadiamento: T4NOMx). A paciente apresentou boa evolução no pós-operatório, e com 18 meses da cirurgia encontra-se sem sinais de recidiva tumoral. Discussão: Os somatostatinomas são tumores raros, geralmente malignos, mais freqüentemente encontrados no pâncreas, sendo que apenas 2,5\% deles são funcionantes. 0 diagnóstico é realizado por exame anátomo-patológico e imuno-histoquímico e o tratamento de escolha é a ressecção cirúrgica. Quando a cirurgia curativa não pode ser realizada, alguns pacientes podem se beneficiar de procedimentos paliativos e/ou quimioterapia. 0 seguimento deve ser realizado a cada seis meses com tomografia abdominal e a dosagem sérica de somatostatina pode ser um método complementar. A sobrevivência em cinco anos após a retirada completa do tumor e na ausência de linfonodos comprometidos ou metástases chega a $100 \%$.
\end{abstract}

Unitermos: Somatostatinoma; tumor carcinóide; tumores neuroendócrinos.

\section{Abstract}

Introduction: Somatostatinomas are rare somatostatin-producing carcinoid tumors, mainly found in the duodenum and pancreatic head. The importance of this case report lies in the rarity of such histological subtype. Case Report: Sixty-eight years old female patient with a two-year diabetes history and fivemonth abdominal pain complaint. Physical examination revealed a tumor in the epigastrium and right hypochondrium, immovable and painful upon palpation. Ultrasound and abdominal computerized tomography showed, respectively, calculous chronic cholecystitis and solid tumour, measuring approximately $12 \mathrm{x}$ $8 \mathrm{~cm}$, located in pancreatic head topography. The patient was submitted to surgery in which a large tumor was found evolving duodenum and pancreas. Cephalic gastroduodenopancreatectomy and cholecistectomy were performed. The anatomopathological and immunohistochemical study of the surgical piece revealed a duodenal somatostatinoma (stage: T4NOMx). The patient afforded good postoperative evaluation and after 18 months from surgery, presented no signs of tumor recurrence. Discussion: Somatostatinomas are rare, usually malignant tumors, more frequently found in the pancreas, and only $2.5 \%$ are functional. Diagnosis is established by anatomopathological and immunohistochemical exams and the chosen treatment is surgical resection. When curative surgery cannot be performed, some patients may benefit from palliative surgery and/or chemotherapy. Follow-up must take place every six months with abdominal tomography and serum somatostatin dosage could be a complementary method. The five-year survival rate is $100 \%$ upon complete tumor resection with the absence of affected lymph nodes or metastasis.

Keywords: Somatostatinoma; carcinoid tumor; neuroendocrine tumors.

Recebido: 25/06/2007

Revisado: 17/03/2008

Aprovado: 24/03/2008

Estudo realizado no Hospital de Ensino Padre Anchieta, São Bernardo (SP), Brasil

Disciplina de Cirurgia do Aparelho Digestivo da Faculdade de Medicina do ABC (FMABC), Santo André (SP), Brasil

2 Disciplina de Patologia Geral e Especial da FMABC

Endereço para correspondência: Alexandre Cruz Henriques, Rua Mediterrâneo, 928 - Jardim do Mar, CEP $09750-420$ - São Bernardo do Campo (SP)

Tel: (11) 41259177,E-mail: achenriques@uol.com.br 


\section{Introdução}

Tumores carcinóides são neoplasias neuroendócrinas e represen$\operatorname{tam} 2 \%$ de todos os tumores malignos do sistema digestivo ${ }^{1-3}$. No trato gastrintestinal originam-se de células enterocromafins que podem se manifestar com diferentes achados clínicos e anátomo-patológicos, dependendo do local de origem e do hormônio que secretam ${ }^{4-5}$. Somatostatinomas são tumores carcinóides raros produtores de somatostatina, localizados principalmente na cabeça do pâncreas e duodeno $0^{6-7}$.

Devido a raridade desse subtipo histológico e ao fato do tumor encontrado possuir grandes dimensões e ausência de metástases linfáticas ou à distância na época do diagnóstico, relata-se o presente caso.

\section{Relato do caso}

Mulher, 68 anos, negra, com queixa de dor epigástrica há cinco meses, de moderada intensidade, com irradiação para ambos os hipocôndrios, e que melhorava com uso de analgésicos. Referia diabetes mellitus há dois anos e hipercolesterolemia.

O exame físico do abdome evidenciava massa em epigástrio e hipocôndrio direito, fibroelástico, fixo, superfície lisa, limites imprecisos, dor à palpação e extensão de cerca de 10 x $6 \mathrm{~cm}$.

A ultra-sonografia e a tomografia computadorizada do abdome visibilizaram, respectivamente, colecistopatia crônica calculosa e massa sólida de contornos lobulados medindo cerca de $12 \times 8 \mathrm{~cm}$, localizada em topografia de cabeça de pâncreas e sem plano de clivagem com a mesma (Figura 1).

Com hipótese diagnóstica de tumor de cabeça de pâncreas e colelitíase, foi indicada laparotomia exploradora, não sendo solicitadas dosagens hormonais como complementação diagnóstica.

No intra-operatório foi encontrado tumor volumoso situado na face posterior do bloco duodenopancreático, sem plano de clivagem com o mesmo (Figura 2). Optou-se pela realização de gastroduodenopancreatectomia cefálica, com reconstrução em alça única e colecistectomia.

O exame anátomo-patológico da peça cirúrgica revelou neoplasia em porção descendente de duodeno, comprometendo toda a parede do órgão e comprimindo o tecido pancreático adjacente. As margens cirúrgicas e nove linfonodos loco-regionais isolados estavam livres de comprometimento neoplásico (estadiamento: T4N0Mx). Havia ainda colecistite crônica calculosa.

O quadro histopatológico e imuno-histoquímico foi compatível com neoplasia de padrão neuroendócrino (tumor carcinóide), com imunoexpressão positiva para cromogranina e somatostatina e negativa para glucagon e insulina, sendo, portanto diagnosticado como um somatostatinoma (Figura 3).

A paciente apresentou boa evolução pós-operatória e recebeu alta no décimo dia de pós-operatório. Após 18 meses da operação, a paciente permanece assintomática e sem evidências de recidiva tumoral.

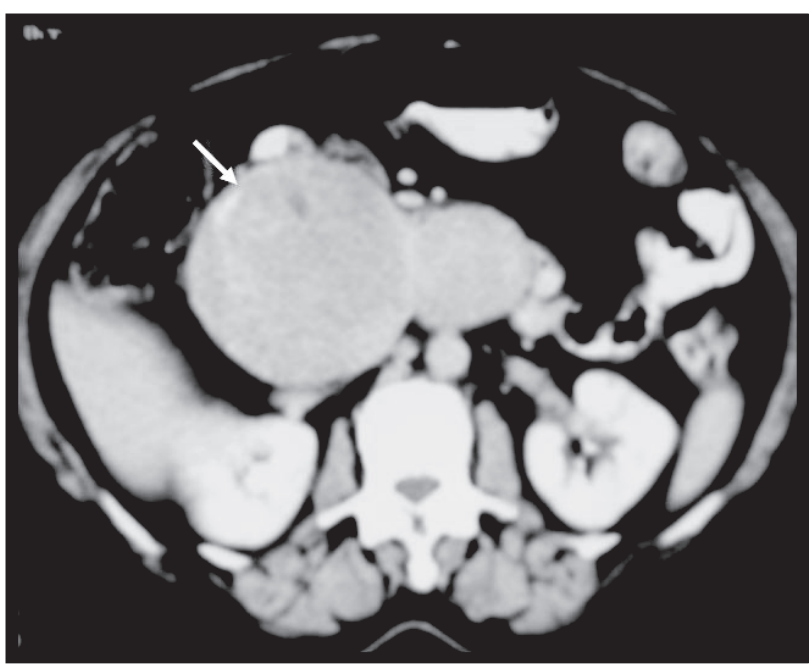

Figura 1 - Tomografia abdominal - tumor de aproximadamente $12 \times 8 \mathrm{~cm}$, contornos lobulados, localizado em topografia de cabeça de pâncreas (seta).

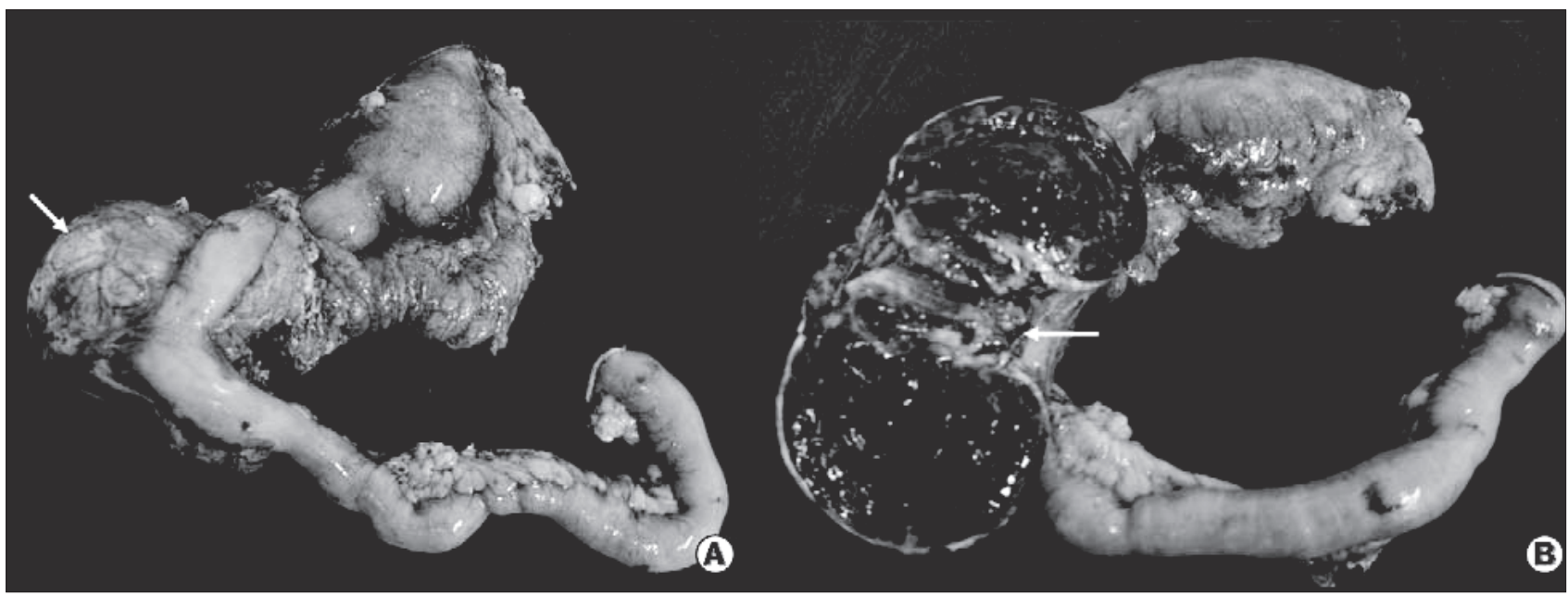

Figura 2 - Peça cirúrgica - (A) Tumor localizado na porção descendente do duodeno (seta); (B) Corte (seta) demonstrando aspecto macroscópico heterogêneo. 


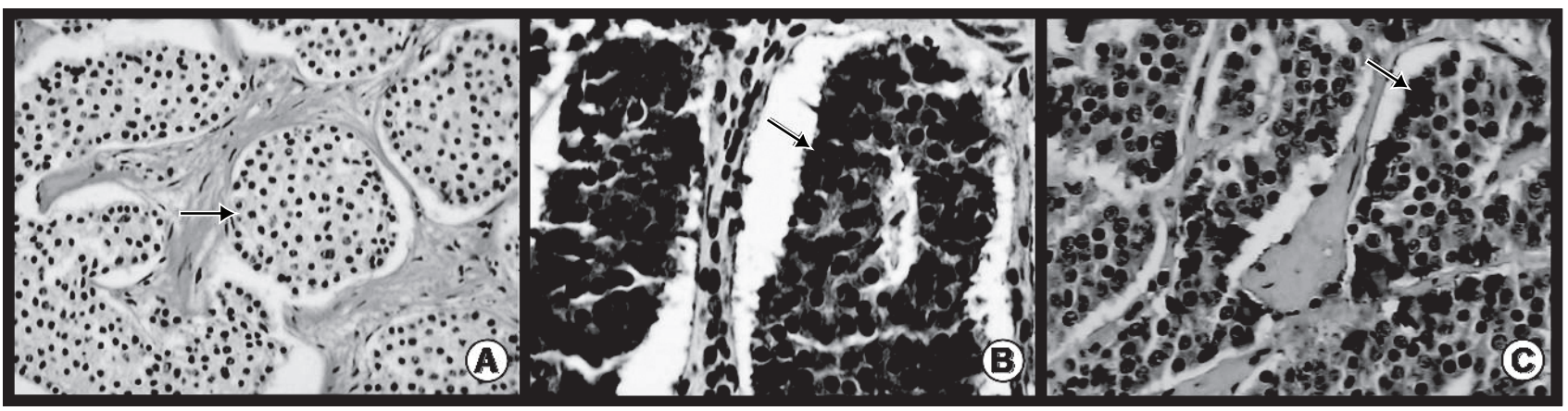

Figura 3 - Fotomicrografias - (A) Células neoplásicas dispostas em blocos (seta), separados por septos fibrosos bem vascularizados, com citoplasma claro, baixo pleomorfismo e aspecto monótono, sugerindo diferenciação neuroendócrina (hematoxilina e eosina - 100x); (B) Forte positividade das células neoplásicas para cromogranina (seta), confirmando a histogênese neuroendócrina (imuno-histoquímica - 400x); (C) Presença de imunoexpressão positiva para somatostatina em grande número de células neoplásicas (seta), configurando o diagnóstico de somatostatinoma (imuno-histoquímica - 400x).

\section{Discussão}

A primeira descrição de somastostatinoma pancreático foi em $1977^{8}$, enquanto que o primeiro caso desse tumor localizado no duodeno data de $1979^{9}$.

Os somatostatinomas são tumores carcinóides raros produtores de somatostatina, que representam menos de $1 \%$ de todas as neoplasias endócrinas gastroenteropancreáticas ${ }^{1,10-11}$, com incidência anual estimada em 1:40 milhões de pessoas 5 . A média de idade dos pacientes acometidos é de 54 anos $^{5}$, e raramente ocorre antes dos 25 anos $^{12}$. Somatostatinomas pancreáticos são mais freqüentes no sexo feminino, enquanto que os extrapancreáticos ocorrem preferencialmente no sexo masculino ${ }^{5}$. Dois terços dos somatostatinomas são originados do pâncreas, sendo o segundo lugar mais freqüente o duodeno. Neste, a região mais acometida é a descendente ( $60 \%$ na área periampular $)^{11-13}$, como ocorreu no presente relato, e no pâncreas, a porção cefálica do órgão é a mais envolvida ${ }^{13}$.

Os somatostatinomas são freqüentemente malignos $(90 \%)^{12}$ e cerca de 30\% dos duodenais e 70\% dos pancreáticos apresentam metástases já no diagnóstico ${ }^{13}$, habitualmente no fígado (40\%) e linfonodos $(36 \%)^{11-12}$. Os somatostatinomas pancreáticos são, em geral, maiores que os duodenais, com tamanho médio variando entre 0,3 e $6 \mathrm{~cm}^{11,14}$.

Os somatostatinomas duodenais manifestam-se freqüentemente com dor abdominal (25\%), icterícia (25\%) e colelitíase $(19 \%)^{11}$. Entretanto, apenas $2,5 \%$ deles são funcionantes ${ }^{6}$. Os não funcionantes apresentarão sintomas relacionados ao espaço ocupado pelo tumor, como obstrução pancreática ou intestinal ${ }^{5}$.

Níveis elevados de somatostatina podem ser constatados principalmente nos tumores maiores do que $4 \mathrm{~cm}$ de diâmetro ${ }^{5}$ e desencadear hiperglicemia, má absorção (diarréia ou esteatorréia) e colelitíase, conhecida como tríade "inibitória" do somastostatinoma; as duas primeiras devido à inibição da atividade secretória endócrina e exócrina pancreática e a última por supressão da motilidade da vesícula biliar; todos sintomas causados pelo excesso de somatostatina ${ }^{1,11,13}$. O tumor de pâncreas cursa com essa síndrome (total ou parcial) em 18,5\% das vezes, enquanto que o de duodeno tende a ser não funcionante ${ }^{13}$. É tam- bém conhecida a associação entre os somatostatinomas e a doença de Von Recklinghausen ou neurofibromatose tipo-I, desordem genética, caracterizada por áreas hiperpigmentadas na pele (manchas “café-comleite”) e neurofibromas cutâneos ${ }^{11,13}$.

O diagnóstico pode ser feito por endoscopia digestiva alta seguida de biópsia, exame anátomo-patológico e imuno-histoquímico, quando o tumor for localizado no duodeno 5 . A tomografia computadorizada, a ressonância nuclear magnética e a ultra-sonografia podem revelar massa tumoral pancreática que, associada a um aumento do nível sérico de somatostatina maior que $2,5 \mathrm{pg} / \mathrm{mL}$, sugerem o diagnóstico de somatostatinoma ${ }^{3,5,13}$. Pode ser também utilizada a ultra-sonografia endoscópica como exame complementar, útil na avaliação da extensão local da lesão e do acometimento linfonodal loco-regional ${ }^{15}$.

Os somatostatinomas são em geral bem localizados radiologicamente: apresentam-se por nódulos solitários e com diâmetro médio de $5 \mathrm{~cm}^{5}$, entretanto, suas manifestações clínicas são variáveis e a raridade desses tumores ${ }^{16}$ faz com que a maioria seja diagnosticada pelos exames anátomo-patológico e imuno-histoquímico ${ }^{11}$ após ressecção cirúrgica ${ }^{5,13}$, como ocorreu no presente caso.

Após suspeita de tumor carcinóide pelo exame anátomo-patológico, utiliza-se da imuno-histoquímica para caracterizar a natureza neuroendócrina da neoplasia, podendo-se utilizar a cromogranina A, a enolase neurônio-específica e a sinaptofisina como marcadores ${ }^{3}$. O diagnóstico de somatostatinoma, no entanto, é dado pela expressão positiva de somatostatina pelas células tumorais, observadas à imunohistoquímica?

O tratamento de escolha para os somatostatinomas duodenais é a ressecção cirúrgica, devido à malignidade destes tumores e por ser a única opção terapêutica curativa ${ }^{2,5}$. Entretanto, apenas $65 \%$ dos casos operados resultam na completa retirada do tumor ${ }^{5,17}$. A duodenopancreatectomia (cirurgia de Whipple) é a cirurgia mais realizada, por estar o tumor, na maioria dos casos, situado na região periampular ${ }^{5,13}$. Nos tumores menores que $2 \mathrm{~cm}$, a ressecção local é possível ${ }^{13}$.

Alguns pacientes podem se beneficiar com a cirurgia paliativa, visando alívio da dor, desobstruções biliares ou intestinais e controle 
de sintomas pela diminuição dos níveis séricos de somatostatina nas neoplasias funcionantes 5 . Quando mais de $90 \%$ do tumor é retirado, pacientes referem alívio dos sintomas por mais de dois anos ${ }^{5}$. Nos casos em que a cirurgia não é possível, a quimioterapia parece controlar os sintomas e beneficiar o paciente ${ }^{16}$.

Além da impossibilidade de ressecção cirúrgica local ou regional completa do tumor ${ }^{17}$, outros fatores parecem também reduzir a sobrevivência nos pacientes com somatostatinomas, tais como acometimento linfonodal, diferenciação celular, status não funcionante do tumor e doença metastática ${ }^{18-19}$. O mesmo é observado para o ta- manho do tumor, pois em $75 \%$ dos tumores maiores que $3 \mathrm{~cm}$ há a presença de metástases hepáticas ocultas ${ }^{11,18,20}$.

O seguimento deve ser realizado a cada seis meses com tomografia abdominal, e a dosagem sérica de somatostatina pode ser um método complementar ${ }^{12}$

A sobrevivência média, em cinco anos, para os pacientes com somatostatinomas é de aproximadamente $60 \%$. Na presença de metástases hepáticas, esta é de $40 \%$. Porém quando a ressecção é completa e não existem linfonodos comprometidos ou metástases, a sobrevivência chega a $100 \%$ em cinco anos ${ }^{5,6}$.

\section{Referências}

1. Garbrecht N, Anlauf M, Schmitt A, Henopp T, Sipos B, Raffel A, et al. Somatostatin-producing neuroendocrine tumors of the duodenum and pancreas: incidence, types, biological behavior, association with inherited syndromes, and functional activity. Endocr Relat Cancer 2008;15(1):229-41

2. Hoffmann KM, Furukawa M, Jensen RT. Duodenal neuroendocrine tumors: classification, functional syndromes, diagnosis and medical treatment. Best Pract Res Clin Gastroenterol 2005;19(5):675-97.

3. Tomassetti P, Migliori M, Lalli S, Campana D, Tomassetti V, Corinaldesi R. Epidemiology, clinical features and diagnosis of gastroenteropancreatic endocrine tumours. Ann Oncol 2001;12 Suppl 2: S95-9.

4. Maggard MA, O'Connell JB, Ko CY. Updated population-based review of carcinoid tumors. Ann Surg 2004;240(1):117-22.

5. House MG, Yeo CJ, Schulick RD. Periampullary pancreatic somatostatinoma. Ann Surg Oncol 2002;9(9):869-74.

6. Soga J, Yakuwa Y. Somatostatinoma/inhibitory syndrome: a statistical evaluation of 173 reported cases as compared to other pancreatic endocrinomas. J Exp Clin Cancer Res 1999;18(1):13-22.

7. Waisberg J, de Matos LL, Waisberg DR, dos Santos HV, Fernezlian SM, Capelozzi VL. Carcinoid of the minor duodenal papilla associated with pancreas divisum: case report and review of the literature. Clinics 2006;61(4):365-8.

8. Larsson LI, Hirsch MA, Holst JJ, Ingemansson S, Kuhl C, Jensen SL, et al. Pancreatic somatostatinoma. Clinical features and physiological implications. Lancet 1977;1(8013):666-8.

9. Kaneko H, Yanaihara N, Ito S, Kusumoto Y, Fujita T, Ishikawa $S$, et al. Somatostatinoma of the duodenum. Cancer 1979;44(6):2273-9.

10. Jordan PH Jr. A personal experience with pancreatic and duodenal neuroendocrine tumors. J Am Coll Surg 1999;189(5):470-82.
11. Tanaka S, Yamasaki S, Matsushita H, Ozawa Y, Kurosaki A, Takeuchi K, et al. Duodenal somatostatinoma: a case report and review of 31 cases with special reference to the relationship between tumor size and metastasis. Pathol Int 2000;50(2):146-52.

12. Rios A, Fernandez JA, Rodriguez JM, Lujan JA, Martinez E, Parrilla P. Massive upper gastrointestinal bleeding as a manifestation of somatostatinoma of the ampulla of vater. Dig Dis Sci 2001;46(10):2162-5.

13. Patel VG, Henderson VJ, Fairweather DA, Fortson JK, Weaver WL, Martin $\mathrm{DM}$, et al. Malignant duodenal somatostatinoma presenting in association with von Recklinghausen disease. Am Surg 2003;69(12):1077-82.

14. Taccagni GL, Carlucci M, Sironi M, Cantaboni A, Di Carlo V. Duodenal somatostatinoma with psammoma bodies: an immunohistochemical and ultrastructural study. Am J Gastroenterol 1986;81(1):33-7.

15. Mathonnet M. Gastrointestinal carcinoid tumors: a multi-technique diagnostic approach. J Chir (Paris) 2007;144(4):287-92.

16. Fulfaro F, Quagliuolo V, De Conno F, Ripamonti C. Carcinoid somatostatinoma of the duodenum. Eur J Surg Oncol 1998;24(6):601-4.

17. Konomi K, Chijiiwa K, Katsuta T, Yamaguchi K. Pancreatic somatostatinoma: a case report and review of the literature. J Surg Oncol 1990;43(4):259-65.

18. Madeira I, Terris B, Voss M, Denys A, Sauvanet A, Flejou JF, et al. Prognostic factors in patients with endocrine tumours of the duodenopancreatic area. Gut 1998:43(3):422-7.

19. Eriksson B, Oberg K, Skogseid B. Neuroendocrine pancreatic tumors. Clinical findings in a prospective study of 84 patients. Acta Oncol 1989;28(3):373-7.

20. Weber HC, Venzon DJ, Lin JT, Fishbein VA, Orbuch M, Strader DB, et al. Determinants of metastatic rate and survival in patients with ZollingerEllison syndrome: a prospective long-term study. Gastroenterology 1995;108(6):1637-49. 